\title{
LOGRO EDUCACIONAL EN JÓVENES Y CLASE SOCIAL DE ORIGEN: EL CASO DEL GRAN CONCEPCIÓN
}

\author{
RICARDO RIVAS
}

\begin{abstract}
RESUMEN
Este artículo, sintetiza los principales elementos teóricos y empíricos de la construcción de un esquema de clases basado en los principios neoweberianos de John Goldthorpe para luego observar la movilidad educacional de los jóvenes entre 20 y 29 años de hogares pertenecientes a dichas clases sociocupacionales establecidas, desde la base de datos del Censo de Población y Vivienda en Chile del año 2002. Al respecto resaltan resultados como es la presencia de movilidad educacional ascendente en la mayoría de las clases, la alta probabilidad de herencia en padres con ciclo básico y la diferencia en las probabilidades de alcanzar educación superior en cada clase al tener un padre con enseñanza básica y un padre con enseñanza media.
\end{abstract}

\footnotetext{
PALABRAS ClAVE: MOVILIDAD EDUCACIONAL, Clase SOCIOCUPACIONAL, INIQUIDAD EDUCACIONAL
}

* Sociólogo chileno, docente Escuela de Trabajo Social, Universidad San Sebastián, sede Concepción, Chile. E-Mail: rrivas@uss.cl. 


\title{
RESUMO
}

Este artigo sintetiza os principais elementos teóricos e empíricos da construção de um esquema de classes baseado nos princípios neoweberianos de John Goldthorpe, para em seguida observar a mobilidade educacional de jovens de 20 a 29 anos pertencentes a tais classes sociocupacionais estabelecidas a partir da base de dados do Censo de População e Domicílio no Chile no ano 2002. Nesse sentido, ressaltam os resultados de como ocorre a mobilidade educacional ascendente na maioria das classes, a alta probabilidade de herança (educacional) em pais com ciclo fundamental, e a diferença nas probabilidades de se alcançar educação superior em cada classe, tendo-se um pai com ensino fundamental e um pai com ensino médio.

\section{PALAVRAS CHAVE: MOBILIDADE EDUCACIONAL, Classe SOCIOCUPACIONAL, DesiguALDADE EDUCACIONAL}

\begin{abstract}
This article, synthesizes the main theoretical and empirical elements of the construction of a scheme of classes based on the Neoweberian principles of John Goldthorpe, in order to observe the educational mobility of youth between the ages of 20 and 29, who come from homes pertaining to these established socio-occupacional classes, from the data base of the Census of Population and Housing in Chile from the year 2002. To this regard, results stand out as the presence of ascending educational mobility in the majority of the classes, the high probability of inheritance in parents with a basic cycle and the differences in probabilities of reaching for a higher education in each class when having one parent with a secondary education, and the other with only a basic education.
\end{abstract}

KEY WORDS: EDUCATIONAL MOBILITY, SOCIO-OCUPATIONAL CLASS, EDUCATIONAL INEQUITY 


\section{ASPECTOS TEÓRICOS CENTRALES RESPECTO A LA INIQUIDAD EDUCACIONAL}

PODEMOS COMPRENDER LA EQUIDAD desde el punto de vista de las oportunidades de vida que posean los individuos, que cercano a lo que expresara Max Weber, ${ }^{1}$ se puede entender - entre otros factores - como capital educacional que éstos posean como entorno y como capacidades propias. Respecto a la educación como capital individual o capacidades propias, ésta tiene una directa incidencia en la capacidad de alcanzar posiciones ocupacionales de mayor competencia, y por lo tanto, una mejor inserción en el mercado de trabajo. ${ }^{2}$ Es así como «un graduado de la escuela primaria gana un 50\% más que una persona que jamás haya asistido a la escuela, un graduado de la enseñanza secundaria percibe un $120 \%$ más, y un licenciado universitario cobra un 200\% más» (BID 1998, citado por Reimers, 2002).

Sobre el entorno, cabe decir que existen ciertas condiciones inmodificables para los individuos (adscritos) que es, además de las condiciones económicas, el capital educacional de sus padres o de quiénes cumplan dicho rol. Es así como el clima educacional del hogar es aún más determinante del nivel educacional de los hijos que el ingreso familiar (CEPAL 2000; León y Martínez, 2001). La relación entre generaciones respecto al grado de educación alcanzado, nos habla de la igualdad/desigualdad existente en una sociedad. Por un lado, está la cobertura educacional que puede darse en distintos momentos o segmentos sociales, y por otro lado, está el estudio de las posibilidades de alcanzar ciertos umbrales educativos en estos segmentos sociales. ${ }^{3}$

1 Para Weber hay una distinción central que debemos tener en cuenta: la diferencia entre clase y clase social. Una clase es un conjunto de personas que tienen en común ciertas condiciones de oportunidades en base a la posesión de bienes y de formas de generación de ingresos, entre las cuales estaría las cualificaciones y la educación (Crompton, 1997). Sin embargo, éstas no son comunidades, ellas representarían meramente las bases posibles y frecuentes para la acción comunal (Gerth y Mills, en Hamuy ,1958) y por lo tanto así constituirse en clases sociales.

2 Y de esta forma mejor nivel socioeconómico. Al respecto es importante tener en cuenta que «mantener buenas posibilidades de acceder al bienestar implica como mínimo completar el ciclo secundario, o sea alcanzar 12 o más años de estudio en casi todos los casos» (CEPAL, 2004:78).

3 Fernando Reimers (2002) agrega 3 ideas que nos parecen importantes: a) en resumen, la educación constituye una vía potencialmente prome- 


\section{a) La experiencia latinoamericana y chilena respecto a la iniquidad educacional}

En general, en América Latina han existido avances respecto a indicadores educativos básicos como es el alfabetismo y en cobertura educacional en los dos primeros ciclos, sin embargo mantiene niveles evidentemente más bajos que los países de la $\mathrm{OCDE}^{4}$ (Reimers, 2002).

Respecto al traspaso generacional de los niveles educacionales, la CEPAL declara que «los estudios muestran que, en la región, sólo tuvieron movilidad educacional dos de cada cuatro jóvenes urbanos y uno de cada cuatro rurales. En consecuencia, cabe concluir que la probabilidad de movilidad social prácticamente no ha cambiado desde 1980» (CEPAL, 2000:61) Relacionado con el bienestar material, podemos decir que ha existido una correlación entre los indicadores de igualdad educativa y los de bienestar socioeconómico. Es así como la desigualdad educativa en América Latina ha estado en estrecha relación con la distribución de ingresos (CEPAL, 2000; Reimers 2002).

En el caso de Chile podemos ver que «se observa en la enorme brecha educativa por generación. Es así como los que tienen en el año 2002 entre 60 y 64 años alcanzaron en promedio 7.2 años de educación, mientras que los que tienen entre 20 y 24 años ya poseen al menos 11,4 años promedio» (Valenzuela y Herrera, 2003:115).

tedora de movilidad social y un nexo importante en la reproducción de la desigualdad, con lo que coinciden además Torche y Wormald (2004); b) «La influencia de la educación de los padres en el éxito escolar se extiende más allá de su impacto en la disposición escolar en el momento en que los chicos matriculan en los centros educativos... un reciente estudio comparativo sobre 40 países confirma que la instrucción de los padres es uno de los predictores más sólidos de los logros de los alumnos en matemáticas y ciencias»».

4 En el caso de Chile, los niveles de cobertura educacional (tasas netas) ha ido en aumento las últimas décadas, alcanzando niveles considerables en el nivel básico y medio. Así, en base a las encuesta CASEN se obtiene al año 2006 un 36.9\% en educación parvularia, 92.4\% en educación básica, $71.6 \%$ en educación media y un $27.4 \%$ en educación superior. Sin embargo, la brecha entre el promedio de años de estudio en el medio rural y en el urbano ha disminuido débilmente. 
Por otro lado, tomando en cuenta el nivel de escolaridad alcanzado por los hijos respecto al de sus padres en el censo 2002, ${ }^{5}$ 'en el caso de los padres con ciclos educativos medio y básico, se observa cómo estos han logrado que sus hijos accedan a un ciclo más arriba (Valenzuela y Herrera, 2003:117).

Sin embargo, creemos que debemos considerar la observación que hace Torche y Wormald (2004:32) respecto al estudio de la desigualdad educacional: «Si se quiere analizar la igualdad de oportunidades en el ámbito de la educación, entre personas con distinto nivel educacional de origen (educación de los padres) se debe estudiar la movilidad entre diferentes niveles de educación controlando por el cambio en la estructura. Esto es, el grado de apertura de distintos niveles de educación de destino para personas con diferente origen educacional». Esto nos hace ver la movilidad educacional desde una perspectiva relativa, lo que incorpora además la idea de qué segmentos sociales son los que han sido beneficiados por dicha apertura y avance en la cobertura.

Desde un punto de vista de la estructura social, en el caso de Chile existen dos estudios importantes en la relación entre desigualdad educacional y estructura de clases. En el caso de Arturo León y Javier Martínez (2001:27), «la evidencia parece indicar que la educación de los padres es aún más determinante del nivel de educación que alcanzan los hijos que el ingreso familiar. Lo que no deja lugar a dudas sobre cómo se reproducen las oportunidades y las desventajas entre generaciones»». Por otro lado, para Florencia Torche y Guillermo Wormald (2004) «la expansión de las oportunidades educacionales ha sido aprovechada por los diversos segmentos sociales de un modo diferencial».

$5 \quad$ Al respecto cabe citar la siguiente nota de los autores: «Sólo se ha podido comparar con el Censo la escolaridad de los hijos respecto de sus padres cuando éstos conviven dentro de un mismo hogar. Se han seleccionado sólo los hijos entre 20 y 29 debido a que por su edad ya deberían haber completado la enseñanza media más un año de ciclo superior... por lo que probablemente se está sobreestimando dicha movilidad, puesto que los hijos entre 20 y 29 años que viven con sus padres tienen un mayor nivel educativo en comparación con los jóvenes entre 20 y 29 años que no viven con sus padres» (Valenzuela y Herrera, 2003:132). 


\section{b) La particularidad del ciclo educativo superior para el estudio de la movilidad educacional}

Hemos creído necesario tratar brevemente el tema de la movilidad educacional teniendo un ciclo educativo de referencia. Al respecto, es clave entender por ejemplo que «en muchos países el hecho de haber terminado la educación primaria no representa ninguna diferencia en la probabilidad de estar empleado» (Reimers, 2002). Esto está relacionado con lo que se ha llamado «devaluación de la educación», esto es que «conjuntamente con el aumento de la cobertura y del nivel educacional promedio en la sociedad, se produce una devaluación de la educación por cuanto se necesitan más años de estudio para lograr una misma inserción ocupacional y un ingreso equivalente... la devaluación es mayor para quienes parten de una situación de desventaja, lo que constituye un escollo adicional para la movilidad entre generaciones» (CEPAL, 2000:93).

Por otro lado, si bien la desigualdad educacional entre distintos segmentos sociales e incluso internacionales comienza a darse en el ciclo medio, es el ciclo superior el que muestra la más alta desigualdad en términos de estratos socioeconómicos y en términos de clases. ${ }^{6} \mathrm{Al}$ respecto cabe señalar, que según Larrañaga (2002, citado en PNUD, 2006) una de las causas del marcado contraste en el acceso a la universidad es la altísima correlación entre nivel socioeconómico y puntajes en las pruebas de selección universitaria. ${ }^{7}$

Como nos señala el trabajo de Valenzuela y Herrera (2004) quienes construyeron un indicador socioeconómico tomando en cuenta una serie de variables de capital humano y material - entre otras - de los hogares chilenos comprendidos en el Censo de 1992 y 2002 en Chile,

6 Delia Langa (2004) expone que «Shavit y Blossfeld (1993) marcaron un hito en este sentido cuando mostraron cómo las diferencias en las oportunidades de ir a la universidad según clase social no habían experimentado notables cambios a lo largo del siglo XX, salvo en los casos de Suecia, y en menor medida, Holanda. A su vez Carabaña (2004) llega a la misma conclusión, a partir de una encuesta internacional realizada en 19 países (IALS)». Esta encuesta se realizó en los países de la OCDE, más Chile el año 1998.

7 Otro dato interesante son por ejemplo que según González (2000, citado en PNUD, 2006) las probabilidades que un estudiante de colegio municipalizado ingrese a la educación superior son de un quinto en relación con las de uno proveniente de colegio particular pagado. 
se puede apreciar que el crecimiento del porcentaje de personas de 25 o más años con educación superior del quintil socioeconómico más bajo fue de un $1.6 \%$, en cambio en el quintil socioeconómico más alto fue de un $9.4 \%$. Desde datos más recientes, vemos que en el quintil más pobre, pasó de una tasa de asistencia neta de un 4\% en 1990 a un $14 \%$ en 2006. Sin embargo, este incremento en el quintil más rico fue de un 33\% a un 53\% (Mideplan, 2006).

\section{LA CONSTRUCCIÓN DE UN ESQUEMA DE CLASES}

Antes de continuar directamente con la movilidad educacional, debemos establecer nuestras categorías de observación y comparación. Como adelantáramos, hemos establecido 8 clases sociocupacionales para cumplir con tal propósito.

Cabe tener claro que en un trabajo que se centre en una perspectiva sociológica de «clases», que éstas son categorías nominales que debieran describir, a la vez, entidades o colectivos identificables, y que al menos condicionan la acción colectiva de los sujetos. Desde esta premisa, una distinción es fundamental, y es que por un lado podemos tener estas clases - clases sociocupacionales en nuestro caso- como lo que queremos explicar en su conformación (fenómeno dependiente) o lo que nos explica ciertas diferencias (fenómeno independiente), lo que Carabaña (1997) catalogará la clase como «explanandum» en el primer caso y clase como «explanante» en el segundo.

En este trabajo, se definieron 8 clases sociocupacionales como «explanante» del fenómeno movilidad educacional. Dicha clasificación, se ha basado en el trabajo del británico John Goldthorpe, quien es considerado como uno de los exponentes de la perspectiva neoweberiana de clases.

\section{a) Aspectos centrales de la perspectiva weberiana}

Para Weber las relaciones entre las personas hacen que existan formas de distribución desigual de ciertos bienes tangibles e intangibles, que tiene como producto clases positiva o negativamente privilegiadas. Estos pueden ser bienes de producción pero también de educación y cualificación que configuran «situaciones de clase» en el mercado que tienen como correlato «estructuras de oportunidades».

Si nos centramos en que Weber reconozca que no sólo en el ámbito de la producción se originan desigualdades estructuradas, pode- 
mos relevar los aportes que hace esta premisa en el ámbito del estudio de las clases:

i) No sólo en cómo se relacionan los individuos con la propiedad de los medios de producción que conforma conglomerados de individuos de condiciones objetivas similares, sino además la inserción de éstos en el mercado de trabajo principalmente.

ii) Como consecuencia de lo anterior, la calificación y la educación juegan un rol importante en la configuración de las «portunidades de vida».

iii) Da una importancia al status y al prestigio como una fuente de diferenciación social, quitándole la exclusividad otorgada a las clases en el sentido que le da Marx. En este aspecto, podemos encontrar las raíces para la perspectiva funcional de la estratificación.

iv) Del punto anterior, la dominación no necesariamente está ligada a un fenómeno de explotación económica, sino también a fenómenos de prestigio y poder.

\section{b) El esquema de clases de John Goldthorpe}

John Goldthorpe ha desarrollado su trabajo teórico y empírico a través de estudios en sociedades avanzadas, especialmente en Gran Bretaña. ${ }^{8}$ Sin embargo, ha tenido una influencia en el estudio de clases al menos en Chile y Argentina. ${ }^{9}$

Para Goldthorpe la ocupación es un indicador de la situación de clase de los individuos, más que estrictamente las relaciones de propiedad (González, 1992). Es por eso que integra aspectos weberianos a su trabajo que son además de la propiedad, el conocimiento (Feito, 1997; Martínez, 1999). Además, hace una crítica a cómo se ha trabajado la estratificación social y el estudio de clases desde la ocupación,

8 Perteneciente al grupo de movilidad social del Nuffield Colleg fundado en 1969. Luego junto a Hope construyen la conocida escala de prestigio ocupacional Hope-Goldthorpe. En los setentas se centra en estudios de Movilidad Social en Gran Bretaña. En los ochentas surge el Proyecto CASMIN (Comparative Analisys of Social Movility in Industrial Nations) donde se consolida en estudios transnacionales de estructura de clase y movilidad social (Hernández de Frutos, 1997; Alonso, 1997; Crompton, 1997).

9 En Chile podemos citar el trabajo de León y Martínez (1987 y 2001), León y Tironi (1985) y Torche y Wormald (2004). En el caso de Argentina principalmente el trabajo de Jorrat (2000) y Gómez (2003). 
en concreto a la escala NORC, ${ }^{10}$ que se fundamenta en la perspectiva estructural funcional. (Alonso, 1997).

Basándose en el trabajo de David Lockwood, Goldthorpe desarrolla un esquema de posiciones de clases a partir de las ocupaciones, bajo la óptica y la combinación de (Crompton, 1997): i) Situación de mercado: fuentes y niveles de ingreso, seguridad económica y oportunidades de progreso económico o ascenso social. ii) Situación de trabajo: cómo se insertan en los procesos de producción en cuanto a autoridad dentro de este y grado de control de dicho proceso. iii) Estatus de empleo: en términos de autonomía con la que se inserta en el proceso productivo. En esta parte podemos integrar además la distinción manual-no manual.

Para Goldthorpe, lo importante no es que tan exhaustivo sea un esquema de clase, sino el grado de 'identidad demográfica que alcance' en una formación social específica. Por otro lado, los límites de estas posiciones de clase no están dados por una cualidad ontológica $a$ priori, sino el rendimiento que presente en las pautas de movilidad social de los sujetos, y de qué manera un grupo respecto a otro pueda explicar un ascenso o descenso en esta movilidad. ${ }^{11}$

De esta forma la movilidad social, permite evidenciar: i) El grado de permeabilidad de una sociedad entre estratos o clases según sea el enfoque, y en el contexto de desigualdad correspondiente. ii) De qué manera en las sociedades notoriamente desiguales cierto grado de movilidad social hace que sea una «válvula de escape» (Parkin, 1972, en Crompton, 1997) para aquellos que presentan capacidades de ascenso. iii) Desde un enfoque de formación de clases, las clases bajas se quedan sin sus elementos más capaces, disminuyendo las posibilidades de liderazgos.

Basados en estos principios, desde el Censo de Población y Vivienda en Chile del año 2002, se identificaron las siguientes clases sociocupacionales para los jefes y jefas de hogar en el Gran Concepción: ${ }^{12}$

10 Escala de Prestigio Ocupacional elaborada por North y Hatt en 1947 para el Nacional Opinión Research Center (NORC) de Chicago (Alonso, 1997:113).

11 También debe tenerse en cuenta, que los estudios empíricos de Goldthorpe en Gran Bretaña, decantan en diagnósticos de la situación sociopolítica, esto es, una de las finalidades de sus estudios también poseen una motivación politológica.

12 En promedio, por cada una de las clases sociocupacionales, se trabajó con el $30 \%$ de los hogares. Ya que eran éstos los que figuraban con hijos de 20 a 35 años de edad. Tal como lo revisáramos en nuestro mar- 


\section{CUADRO 1: CATEGORÍA OCUPACIONAL CIUO-88 A DOS DÍGITOS Y VALORES DE SITUACIÓN EN EL EMPLEO QUE CONFORMAN CADA CLASE SOCIOCUPACIONAL BASADO EN GOLDTHORPE Y ERIKSON 1993 (TORCHE Y WORMALD, 2004)}

\begin{tabular}{|c|c|c|c|}
\hline & $\begin{array}{c}\text { Clases basados en } \\
\text { Goldthorpe }\end{array}$ & $\begin{array}{c}\text { Categoría ocupacio- } \\
\text { nal (CIUO-88) }\end{array}$ & Situación en el empleo \\
\hline 1 & Clase de servicio & $\begin{array}{c}21222324111213 \\
3132\end{array}$ & TODAS \\
\hline \multirow[t]{2}{*}{2} & \multirow{2}{*}{$\begin{array}{l}\text { Clase de rutina } \\
\text { no manual }\end{array}$} & 4142 & $\begin{array}{l}1 \text { Trabajador asalariado. } \\
2 \text { Trabajador de servicio doméstico. } \\
5 \text { Familiar no remunerado. }\end{array}$ \\
\hline & & 52 & $\begin{array}{l}1 \text { Trabajador asalariado. } \\
\mathbf{2} \text { Trabajador de servicio doméstico. }\end{array}$ \\
\hline 3 & Pequeña burguesía & $\begin{array}{c}33344142515271 \\
72737481828391 \\
9293\end{array}$ & 4 Empleador. \\
\hline \multirow{2}{*}{4} & \multirow{2}{*}{$\begin{array}{l}\text { Trabajadores } \\
\text { independientes }\end{array}$} & $\begin{array}{l}33345152717273 \\
74818283919293\end{array}$ & $\begin{array}{l}3 \text { Trabajador por cuenta propia. } \\
5 \text { Familiar no remunerado. }\end{array}$ \\
\hline & & 4142 & 3 Trabajador por cuenta propia. \\
\hline 5 & $\begin{array}{l}\text { Pequeño } \\
\text { propietario agrícola }\end{array}$ & 6162 & $\begin{array}{l}3 \text { Trabajador por cuenta propia. } \\
\mathbf{4} \text { Empleador. } \\
\mathbf{5} \text { Familiar no remunerado. }\end{array}$ \\
\hline \multirow[b]{2}{*}{6} & \multirow{2}{*}{$\begin{array}{l}\text { Técnicos inferiores y } \\
\text { FFAA }\end{array}$} & 1 & TODAS \\
\hline & & 3334 & $\begin{array}{l}1 \text { Trabajador asalariado. } \\
\mathbf{2} \text { Trabajador de servicio doméstico. }\end{array}$ \\
\hline 7 & Obrero calificado & 72737481828351 & $\begin{array}{l}1 \text { Trabajador asalariado. } \\
\mathbf{2} \text { Trabajador de servicio doméstico. }\end{array}$ \\
\hline 8 & $\begin{array}{l}\text { Obrero no calificado y } \\
\text { peón agrario }\end{array}$ & 719193616292 & $\begin{array}{l}1 \text { Trabajador asalariado. } \\
\mathbf{2} \text { Trabajador de servicio doméstico. }\end{array}$ \\
\hline
\end{tabular}

Fuente: elaboración propia bajo la supervisión de Julio Carabaña.

Para la construcción de este esquema de clases sociocupacionales, dadas las limitaciones que presentaba el censo en término de variables disponibles, se aseguró una diferencia en términos de años de escolaridad entre ellas. Las clases que no presentaron diferencia estadística fueron la de obrero calificado y técnicos inferiores.

co de referencia empírico, el ejercicio de analizar la movilidad educacional dentro de los hogares usando como fuente la base de datos censal tiene ciertas limitaciones. La principal es que los hogares con presencia de movilidad educacional estén sobrerepresentados. Reiteramos que este ejercicio ha sido realizado por Valenzuela y Herrera (2003 y 2004) y editado en dos publicaciones del INE. 
GRÁFICO 1: MEDIA DE AÑOS DE ESCOLARIDAD POR CLASE SOCIOCUPACIONAL DEL GRAN CONCEPCIÓN

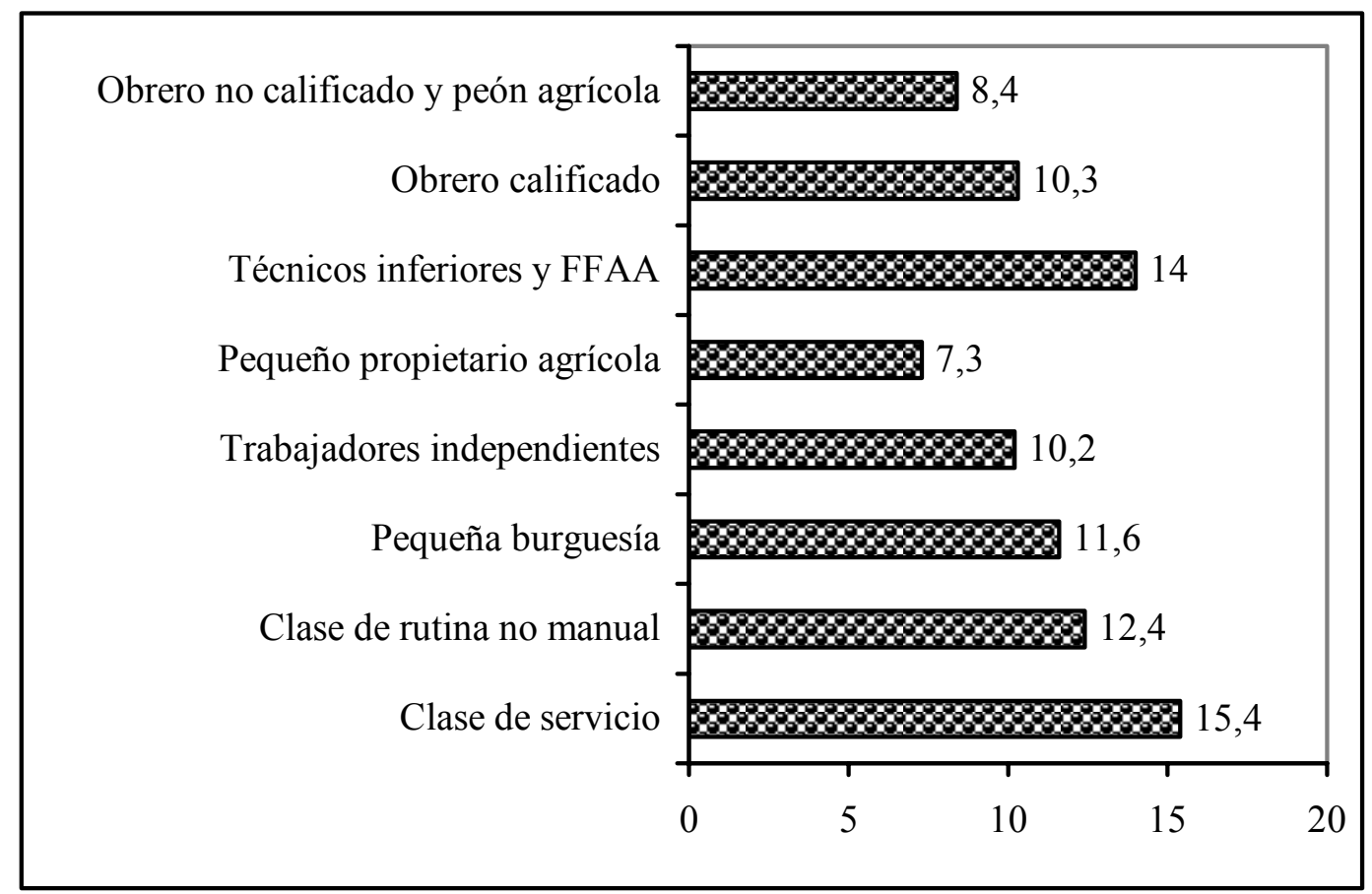

Fuente: base de datos censal INE 2002.

\section{PRINCIPALES RESULTADOS RESPECTO A LA MOVILIDAD EDUCACIONAL}

Tomando en cuenta entonces, la escolaridad del joven en el hogar entre 20 y 29 años respecto a su padre o madre, notamos que el ascenso educacional prevalece en la mayoría de las clases sociocupacionales, siendo la herencia una característica de las clases sociocupacional de Servicio y Técnica. En ninguna se aprecia descenso como categoría modal. Sin embargo, existen dos elementos centrales a la hora de hacer un análisis de la movilidad educacional: Primero, la distancia del «salto» dado debido a este ascenso educacional intergeneracional, donde toma protagonismo el paso de enseñanza media a enseñanza superior. Sin embargo este punto no será central en este trabajo. Y luego, que haya ascenso educacional en la mayoría de los hogares de una determinada clase, no quiere decir que haya más flujo o recambio que en otras, y tampoco oportunidades de movilidad equivalente a las otras. Abordaremos el segundo punto en las siguientes páginas.

Es necesario aclarar que en base a cierta literatura respecto a la movilidad social (Cachón, 1989; Carabaña, 1999; Jorrat 2004), pode- 
mos entender ésta con sus diferentes formas de explorarla a partir del siguiente esquema.

$$
\text { Movilidad Educacional Total } \begin{cases}\text { Absoluta } & \left\{\begin{array}{l}
\text { General } \\
\text { Particular }
\end{array}\right. \\
\text { Relativa } & \left\{\begin{array}{l}
\text { General } \\
\text { Particular }
\end{array}\right.\end{cases}
$$

\section{a) Movilidad absoluta general}

Si en un cuadro de doble entrada, ponemos como columna la escolaridad del padre o madre y como fila la escolaridad de los hijos, podemos dar cuenta desde los porcentajes del total de individuos que están fuera de la diagonal, de la totalidad de casos móviles. Al respecto obtuvimos los siguientes porcentajes.

\section{GRÁFICO 2: PORCENTAJE DE HOGARES POR CADA CLASE SOCIOCUPACIONAL DEL GRAN CONCEPCIÓN EN QUE EXISTE MOVILIDAD EDUCACIONAL}

Obrero no calificado y peón agrícola

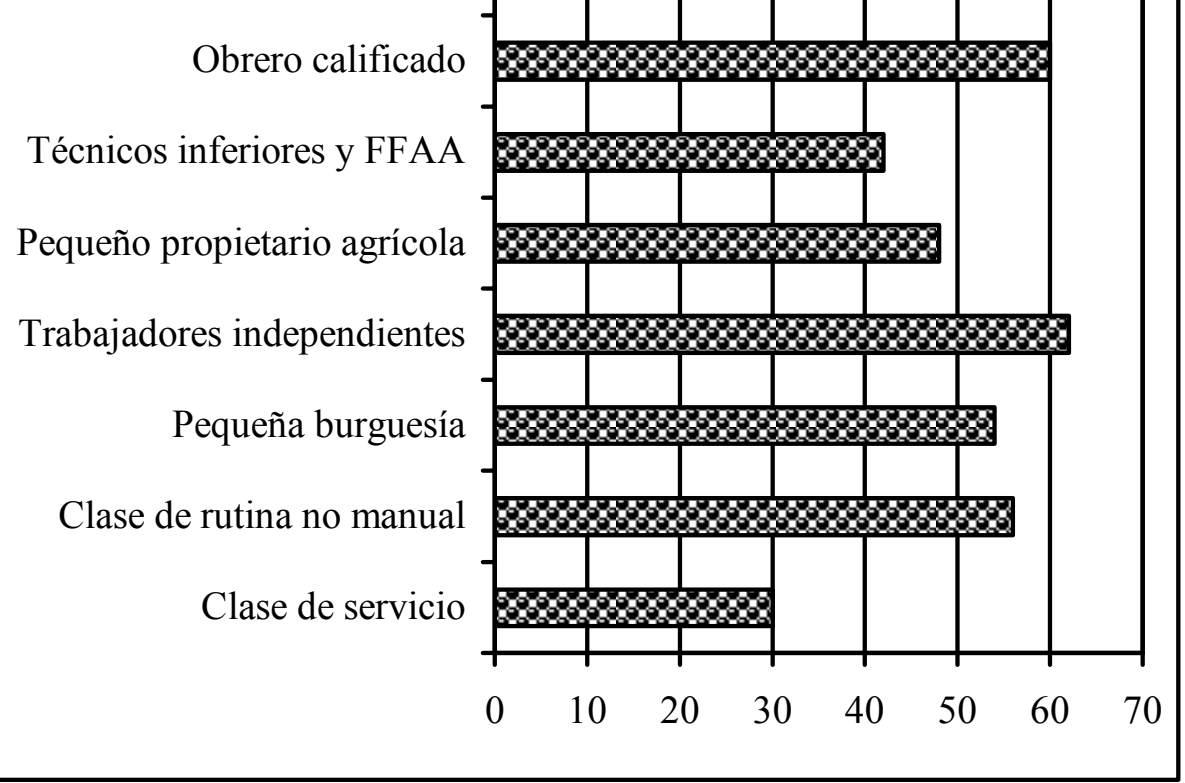

Fuente: base de datos censal INE 2002. 
Vemos que los porcentajes más bajos de movilidad se dieron en las clases de los Técnicos y de Servicio, y en tercer lugar en los pequeños Propietarios Agrícolas. Tomando en cuenta el promedio de escolaridad del padre o madre en cada una de las clases, podemos entender que la mayor herencia de la escolaridad en la clase de Servicio se trata de una herencia de alto nivel educativo, debido al «techo de escolaridad» que posee ésta. En el caso de la clase de Pequeños Propietarios Agrícolas, se trata de una mayor herencia de baja escolaridad, lo que la convierte en una herencia más preocupante en términos de desigualdad.

\section{b) Características de la movilidad educacional relativa}

La movilidad general nos da cuenta de los cambios en volumen de individuos en los distintos ciclos educacionales, por lo tanto si mayores son los cambios en volumen, mayores desplazamientos son los que se requieren. Sin embargo, nada se dice respecto a la igualdad de oportunidades que tienen determinados individuos de cierto origen de ser parte de un proceso de movilidad ascendente, por ejemplo. Una visión general acerca de la movilidad «sería el único aspecto importante de la movilidad si los individuos no se compararan entre sí, lo que se hace o por envidia, o por sentido de la justicia o por ambos» (Carabaña, 1999:31).

El cálculo de oportunidades relativas de los individuos respecto a los de otros orígenes para llegar a ciertos destinos se realiza controlando la movilidad estructural (forzada). El análisis de la movilidad relativa se realiza sobre la base o referente de la movilidad perfecta, esto es que una vez controlado por el cambio en el tamaño de los ciclos educativos de origen y destino, la posibilidades de acceder a cualquier ciclo educativo de destino son totalmente independientes del origen, es decir, son iguales para cualquiera (Wormald y Torche, 2004).

La movilidad perfecta se puede establecer teniendo los valores esperados de cada tabla de contingencia de orígenes y destinos, ésta sería la tabla de referencia. Por lo tanto, la primera de las pruebas a realizar para cada una de estas tablas, por clase sociocupacional, es la del $\mathrm{Chi}^{2}$. Teniendo como hipótesis nula de que no existe una relación significativa entre origen y destino (movilidad perfecta). ${ }^{13}$

13 Para comprender por qué es ésta la hipótesis nula, debemos decir que dado que nos ponemos como modelo heurístico (además normativo) de una sociedad equitativa, el origen educativo de un individuo no debería predecir el destino educativo de éste. 
$\mathrm{Al}$ respecto, en todas las clases el test es significativo $(\mathrm{p}<0.05)$, por lo tanto, ya sabemos que la movilidad no es perfecta, y que existe una relación entre el origen y el destino controlado por los valores marginales. Así, partimos de la base que existe una relación entre origen y destino en cada una de las clases, aunque la fuerza de esta relación sea débil en cada una de las clases. Si continuamos con la idea de que existe una movilidad perfecta (supuesto de independencia), podemos profundizar en las oportunidades de ciertos grupos de individuos de tener un destino educacional ascendente o descendente. Para eso se calcula la razón de movilidad para cada celda de una tabla. A esto Carabaña (1999) le llama movilidad relativa a la media, que consiste en dividir cada valor observado de la celda por su respectivo valor esperado. Esta medida, a juicio de este autor, se debe entender que «si miramos la tabla de movilidad como el resultado de la distribución de algo a los que todos tienen el mismo derecho, entonces el índice de movilidad es una buena medida del privilegio o el agravio de cada categoría en relación a lo que correspondería» (Carabaña, 1999:35).

CUADRO 2: INDICADORES DE MOVILIDAD RELATIVA A LA MEDIA ENTRE NIVEL EDUCACIONAL DEL HIJO Y NIVEL EDUCACIONAL DEL PADRE PARA MUESTRA TOTAL DE HOGARES

\begin{tabular}{|l|c|c|c|}
\hline & Básica y menos & Media & Superior \\
\hline Básica y menos & $\mathbf{2 , 5 4}$ & 1,45 & 0,42 \\
\hline Media & 0,57 & 1,11 & 0,98 \\
\hline Superior & 0,21 & 0,40 & 1,58 \\
\hline
\end{tabular}

Fuente: base de datos censal INE 2002.

Si tomamos en cuenta nuestra muestra sin dividirla en clases, ${ }^{14}$ vemos que los valores más altos se encuentran en dos casillas de la diagonal de la matriz, es decir, existe una tendencia general de herencia del nivel educativo de los jóvenes. Por otro lado, podemos ver que llegan más jóvenes - que lo esperado- a la enseñanza media, provenientes de hogares con padres que han alcanzado dicho nivel de escolaridad o básico. Llama la atención el alto nivel, por sobre lo esperado, de hijos de padres con ciclo básico que se quedan en dicho ciclo, que es el nivel de mayor inercia, provocando altas probabilidades de herencia.

14 No trabajaremos este tipo de cálculo de movilidad por cada una de las clases debido a que extendería demasiado esta exposición de resultados. 


\section{c) Movilidad relativa respecto a otra categoría}

Tal como expresáramos en nuestro apartado de antecedentes, es importante para nuestros fines tener como referencia un ciclo educativo, que en nuestro caso ha sido el ciclo superior.15 Ya no trabajamos bajo la referencia de valores esperados, sino otra categoría. Una ventaja de esta forma de analizar la movilidad - educacional en este caso - es que «los efectos de la composición se dejan de lado» (Carabaña, 1999).

GRÁFICO 3: PORCENTAJE DE POSIBILIDADES DE LLEGAR A LA EDUCACIÓN SUPERIOR DE HIJOS DE PADRES CON ENSEÑANZA BÁSICA

Y MEDIA DE CADA UNA DE LAS CLASES SOCIOCUPACIONALES, RESPECTO A LOS HIJOS DE PADRES CON ENSEÑANZA SUPERIOR

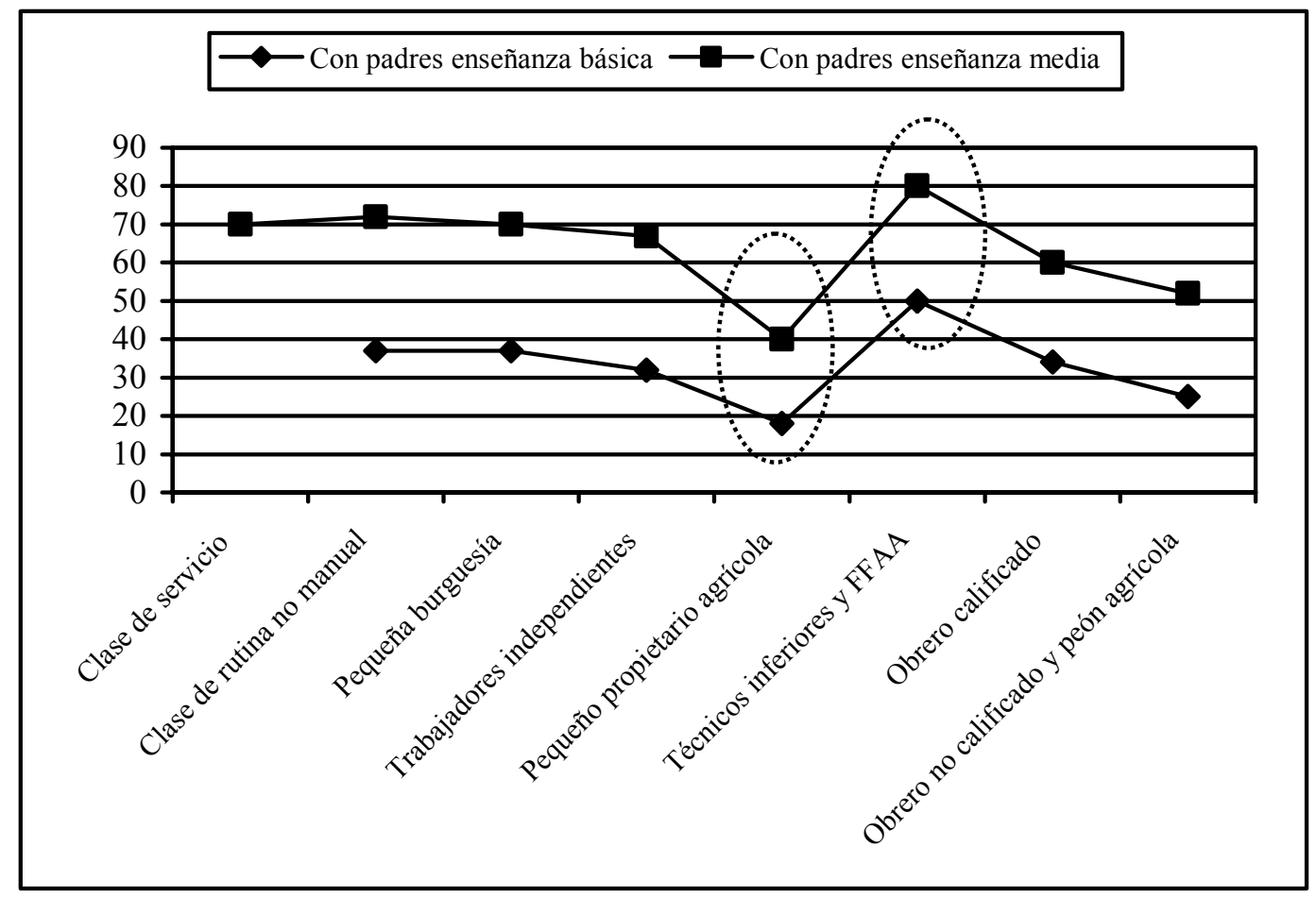

Fuente: base de datos censal INE 2002.

Como reconociéramos anteriormente, la cobertura de la educación superior ha crecido notablemente en los últimos 15 años, manteniendo eso sí, la diferencia en la tasa de dicho crecimiento entre los sectores

15 Hemos elegido este ciclo, ya que de acuerdo a nuestra revisión bibliográfica, es en este ciclo donde más encontramos datos de desigualdad entre estratos que se quieran trabajar. 
de más bajo y más alto ingreso. Sin embargo, nos interesa revisar la desigualdad al respecto teniendo como centro sujetos colectivos, que en nuestro caso son las clases sociocupacionales.

El siguiente sintetiza el porcentaje de oportunidad de alcanzar la educación superior por parte de los hijos de padres con ciclo básico y medio, respecto a las posibilidades que tienen de llegar a este ciclo los hijos de padres que ya lo poseen.

Vemos que en la distribución de los porcentajes de posibilidad de llegar al ciclo superior hay un desplazamiento de curvas, esto es, que ambas siguen una trayectoria similar pero en forma paralela. Desde ya vemos que para todas las clases, que los jóvenes con origen de enseñanza básica, poseen menos posibilidades de llegar al ciclo superior que los que tienen origen en enseñanza media.

Dentro de cada distribución, los que tienen origen de enseñanza básica, la posibilidad más alta es para los hijos pertenecientes a la clase de «técnicos inferiores y FFAA», pues ellos tendrían la mitad de las posibilidades de llegar al ciclo superior (52\%) respecto a los hijos de padres con dicho ciclo. Estas posibilidades aumentan a un $81 \%$ si en esta clase los padres tienen ciclo medio.

En otro extremo, en la clase de «pequeño propietario agrícola» los hijos de jefes de hogar con ciclo básico tienen un $17 \%$ de las posibilidades que posee un hijo con origen en ciclo superior de llegar a dicho ciclo. Ahora, si el jefe de hogar de esta clase tiene ciclo medio, las posibilidades de los hijos aumentan a un 39\% respecto a los hijos de jefes de hogar con ciclo superior de llegar a dicho ciclo.

Si tenemos en cuenta el porcentaje de posibilidades de la muestra total, vemos que la los hijos en la clase de «pequeño propietario agrícola» y «obrero no calificado y peón agrícola» tienen porcentajes de posibilidad más bajos, en ambos ciclos de origen, de llegar al ciclo superior.

Respecto al aumento de posibilidades al tener un padre con ciclo educacional medio en vez de básico es aproximadamente de un $30 \%$ para todas las clases, excepto la clase de «pequeño propietario agrícola», que aumenta sólo en un $20 \%$. 
GRÁFICO 4: PORCENTAJE DE AUMENTO DE POSIBILIDADES DE LLEGAR AL CICLO SUPERIOR DE UN HIJO DE PADRE CON CICLO BÁSICO AL PASAR A UN PADRE CON CICLO MEDIO POR CADA UNA DE LAS CLASES SOCIOCUPACIONALES

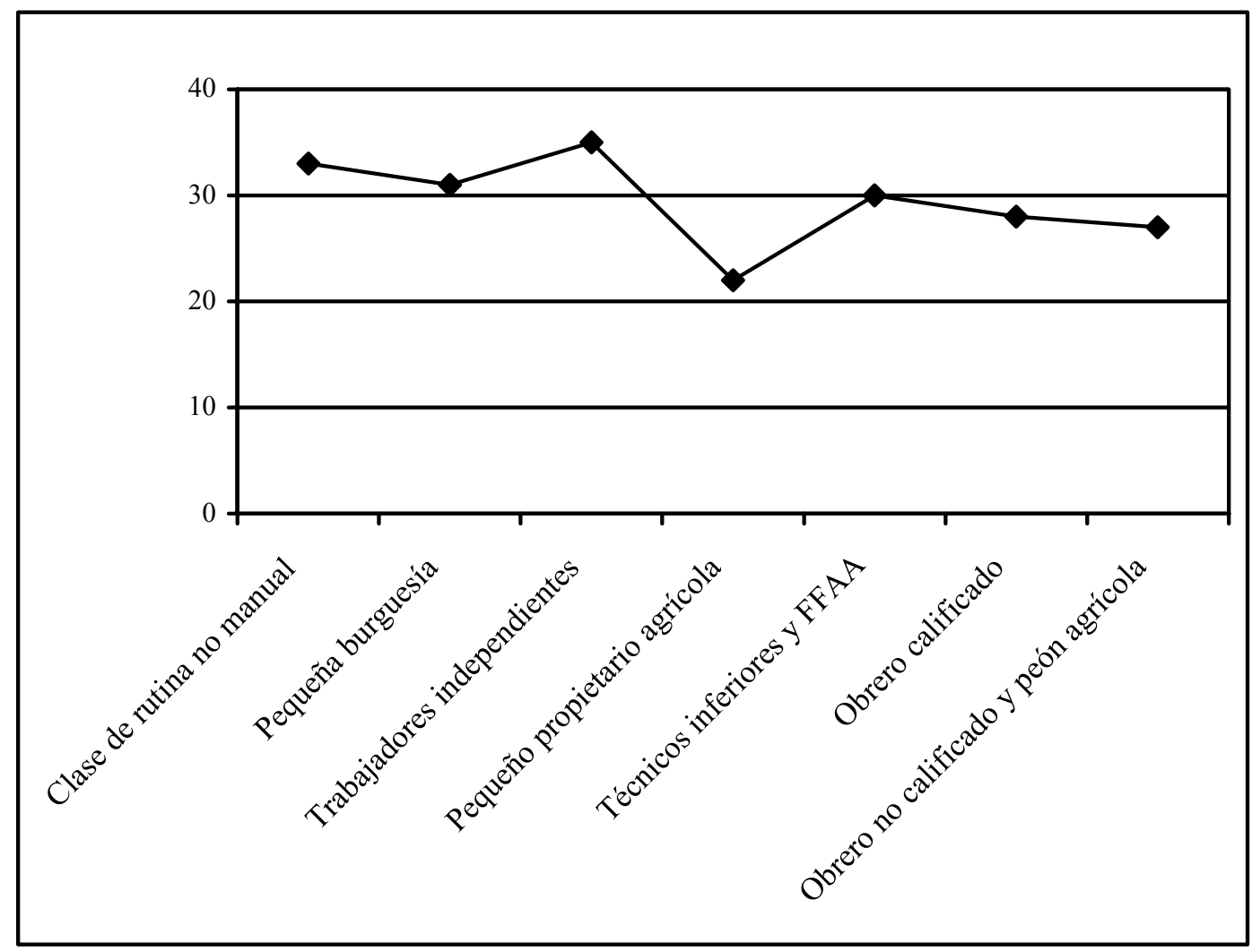

Fuente: base de datos censal INE 2002.

\section{CONCLUSIÓN}

Los jóvenes entre 20 y 29 años fueron parte de un proceso ampliado de movilidad educacional ascendente respecto a la educación de su padre o madre en la mayoría de los hogares de las 8 clases sociocupacionales, a excepción de los hogares donde no había más posibilidades de «subir de nivel educacional», como fueron los pertenecientes a la «clase de servicio» y la clase de «técnicos inferiores y FFAA». Este hallazgo de movilidad educacional generalizado coincide con los de Valenzuela y Herrera (2004) acerca de los mejores niveles educativos alcanzados por las nuevas generaciones respecto a las anteriores en Chile, al menos en la última década. Sin embargo, el análisis de clase y la consideración del origen educacional de los jóvenes nos hace ver que el avance educativo inetergeneracional mantiene ciertas diferen- 
cias, coincidiendo con el planteamiento de Torche y Wormald (2004). Lo puntos que más aportan al la discusión son:

i) Los padres con enseñanza básica son los que menos han traspasado a sus hijos su ciclo educativo, excepto en las clases de «obrero no calificado y péon agrario» y «pequeño propietario agrícola» donde se observan mayor porcentaje de herencia por parte de sus hijos de este ciclo. Por otro lado, viendo la distribución global, las posibilidades de mantenerse en este ciclo son de casi tres veces que los hijos de padres con enseñanza media o superior.

ii) Los padres con enseñanza media traspasan en mayor medida su ciclo educacional que los de ciclo básico. Los porcentajes más altos de este traspaso se da en las «clases obreras»y de «pequeño propietario agrícola».

iii) Los padres con ciclo superior, en general traspasan su ciclo educacional en cerca de un $80 \%$ a sus hijos. Las excepciones son aquellos jefes de hogar que poseen este ciclo pero que pertenecen a las «clases obreras», donde existe una proporción de descenso educacional mayor.

Por otro lado, coincidiendo con Langa (2004) y PNUD (2006) la posibilidad de alcanzar el ciclo superior está marcadamente condicionado por:

iv) Las posibilidades de llegar al ciclo superior de un hijo con padres que poseen los dos ciclos inferiores se diferencia entre las clases, lo que nos habla de una influencia de pertenencia de clase sobre el origen educacional.

v) Si nos centramos sólo en la influencia del origen educacional, vemos que en general que las posibilidades de llegar al ciclo superior aumentan cerca de un 30\% si el padre en vez de tener enseñanza básica, tiene enseñanza media. Este aumento de posibilidades es muy similar para todas las clases. Sin embargo este aumento no es tal en la clase de «pequeño propietario agrícola», que es cerca del $20 \%$.

En general podemos decir que existe una relación estadísticamente significativa entre el nivel educacional del padre respecto al alcanzado por el hijo en cada una de las clases. Sin embargo, se trata de relaciones débiles. Además, podemos coincidir con Atria (2004) que a pesar de la expansión de los sistemas educacionales, las brechas se van 'acomodando' a dicho fenómeno. Cuando los estratos inferiores alcanzan ciertos niveles históricamente mayores de educación, los estratos altos po- 
seen umbrales mayores al respecto. El carácter relacional de las clases sociales puede evidenciar el fenómeno de suma cero en cuanto a las ventajas y desventajas intergeneracionales respecto a la educación.

Finalmente, la desigualdad educacional puede mostrarnos un ámbito de la «desigualdad de partida» de parte de los jóvenes, al momento de considerar la educación como una herencia. Sin embargo, cabe reconocer los avances en términos no sólo de cobertura, sino de movilidad en lo que respecta al ciclo básico en medio, por lo que es el ciclo superior el que presenta hoy los desafíos de equidad más serios, sobre todo si pensamos que es en este ciclo, donde realmente puede haber una movilidad consistente en términos de ingresos y de condiciones generales de vida.

CONCEPCIÓN (CHILE), OCTUBRE 2007

RECIBIDO: OCTUBRE 2007

ACEPTADO: MARZO 2008

\section{REFERENCIAS BIBLIOGRÁFICAS}

Alonso, M., C. Tomé y M. SAZATORnil (1997): Clases sociales ¿discurso publicitario? Madrid: Enymion.

ATRIA, R. (2004): «Estructura ocupacional, estructura social y clases sociales». En www.cepal.cl.

CACHÓN, L. (1989): ¿Movilidad social o trayectorias de clase? Madrid: Siglo XXI.

CARABAÑA, J. (2004): «Educación y movilidad social». En www.ucm.es.

- (1999): Dos estudios sobre movilidad intergeneracional. Madrid: Fundación Argentaria. (1997): «Esquemas y estructuras». Revista Crítica de Ciências Sociais No49.

CEPAl (2004): Una década de desarrollo social en América Latina. 19901999. En www.cepal.cl.

(2000): La brecha de la equidad, una segunda evaluación. En www.cepal.cl.

CROMPTON, R. (1997): Clase y estratificación. Una introducción a los debates actuales. Madrid: Tecnos.

Feito, R. (1997): Estructura social contemporánea. Las clases sociales en los países industrializados. Madrid: Siglo XXI.

GómEZ, G. (2003): «Mujeres y hogares en esquemas de estratificación social». Documento inédito.

GonZÁlez, J. (1992): Clases sociales: estudio comparativo de España y la comunidad de Madrid. En www.madrid.org. 
HAMUY, E. (1958): Antología sobre estratificación social. Santiago: Universitaria.

REIMERS, F. (2002): «Oportunidades y políticas educacionales en latinoamérica». En www.plataforma.uchile.cl.

HERNÁNDEZ DE FRUTOS, T. (1997): Para comprender las estructuras sociales. Pamplona: Verbo Divino.

JORRAT, J. (2004): «Aspectos descriptivos de la movilidad intergeneracional de clase en Argentina: 2003-2004». En www.aset.org.ar.

LANGA, D. (2004): «Las experiencias de los universitarios según clase social: de los distintos modos de construir la identidad juvenil en relación con las apuestas educativas». En www.revistapraxis.cl.

LEÓN, A. y MARTínEZ, J. (2001): «La estratificación social chilena hacia fines del siglo XX». En www.cepal.cl.

MARTíNEZ, R. (1999): Estructura social y estratificación. Reflexiones sobre desigualdades sociales. Madrid: Miño y Dávila Editores.

MidePLAN (2006): «Resultados CASEN educación». En www.mideplan.cl.

PNUD (2006): «Expansión de la educación superior en Chile: hacia un nuevo enfoque de la equidad y la calidad». En www.desarrollohumano.cl.

TORCHE, F. y G. WORMALD (2004): «Estratificación y movilidad social en Chile: entre la adscripción y el logro». En www.cepal.cl.

VALENZUELA, E. y S. HERRERA (2004): «Movilidad social en los hogares más vulnerables de Chile. ¿Cómo ha cambiado la vida de los chilenos? Análisis comparativo de las condiciones de vida de los hogares con menor bienestar socioeconómico (Censos 1992-2002)». Santiago: INE.

— y - (2003): «Movilidad residencial y social. ¿Cuánto y cómo cambiamos los chilenos? Balance de una década, Censos 1992-2002». Santiago: INE. 